\title{
A NEW CLASS OF SPECIAL FUNCTIONS IN HIGHER DIMENSIONS
}

\author{
H. LAITH, A. MOHAMMAD, AND H. DUTTA
}

Received 17 September, 2020

\begin{abstract}
We introduce a new class of functions $\mathcal{G}_{f}^{v}$ that are defined via integrals over $\sigma$-finite measure spaces and their kernels are defined in terms of Bessel functions and measurable functions. We prove that for certain values of $v$, the function $\mathcal{G}_{f}^{v}$ is the Fourier transform of the distribution function $d_{f}$.
\end{abstract}

2010 Mathematics Subject Classification: 28B05; 31A10; 44A20; $35 \mathrm{~S} 30$

Keywords: distribution functions, Bessel function, Fourier transform

\section{INTRODUCTION}

Bessel function of first kind, $J_{v}$, is defined to be the solution of the Bessel differential equation

$$
x^{2} \frac{d^{2} y}{d x^{2}}+x \frac{d y}{d x}+\left(x^{2}-v^{2}\right) y=0
$$

see [6], a series formula of Bessel function can be given by

$$
J_{v}(z)=\left(\frac{z}{2}\right)^{v} \sum_{k=0}^{\infty} \frac{(-1)^{k}}{k ! \Gamma(v+k+1)}\left(\frac{z}{2}\right)^{2 k}
$$

In this paper we study a higher dimensional analog of the function

$$
g_{v}(x)=\Gamma(v+1)\left(\frac{2}{x}\right)^{v} J_{v}(x),
$$

where $x \in \mathbb{R}, v>\frac{-1}{2}$ and $J_{v}$ stands for the Bessel function of the first kind, see [1]. Inequalities involving the function $g_{v}$ have been studied by many authors. For instance, Askey [2] wrote Grünbaum's inequality [4] in terms of the function $g_{v}$. we refer the readers to $[1,5]$ for more background information.

For $n \geq 2$, let $\mathbb{R}^{n}$ be the $n$-dimensional Euclidean space and $\mathbb{S}^{n-1}$ denote the unit sphere in $\mathbb{R}^{n}$ equipped with the surface measure d $\sigma$. Let $(X, \mu)$ be a $\sigma$-finite measure space, that is, there exists a sequence of measurable subsets $\left(A_{n}\right)_{n \in \mathbb{N}}$ such that $X=$ 
$\bigcup_{n \in \mathbb{N}} A_{n}$ and $\mu\left(A_{n}\right)<\infty$ for all $n$. Let $f: X \rightarrow \mathbb{C}$ be a measurable function. Now, if $f \in L^{v+1}(X, \mu)$ consider the function

$$
\mathcal{G}_{f}^{v}(\xi)=\frac{1}{|\xi|^{v}} \int_{X}|f(x)| J_{v}(2 \pi|f(x)||\xi|) \mathrm{d} \mu(x),
$$

where $\xi \in \mathbb{R}^{n}, v \geq \frac{-1}{2}$ and

$$
J_{v}(t)=\frac{(t / 2)^{v}}{\Gamma\left(v+\frac{1}{2}\right) \Gamma\left(\frac{1}{2}\right)} \int_{-1}^{1} e^{i t s}\left(1-s^{2}\right)^{v} \frac{\mathrm{d} s}{\sqrt{1-s^{2}}} .
$$

The function $\mathcal{G}_{f}^{v}(\xi)$ is well defined. To see this, we need to recall the following properties of Bessel functions.

$$
\begin{aligned}
& \left|J_{v}(t)\right| \leq C(v) t^{v}, \\
& \left|J_{v}(t)\right| \leq C(v) t^{-1 / 2},
\end{aligned}
$$

where $t>0$ and the constant $C(v)$ depends only on $v$. Thus, by using (1.1) we have

$$
\begin{aligned}
\left|\mathcal{G}_{f}^{v}(\xi)\right| & =\left|\frac{1}{|\xi|^{v}} \int_{X}\right| f(x)\left|J_{v}(2 \pi|f(x)||\xi|) \mathrm{d} \mu(x)\right| \\
& \leq \frac{1}{|\xi|^{v}} \int_{X}|f(x)|\left|J_{v}(2 \pi|f(x)||\xi|)\right| \mathrm{d} \mu(x) \\
& \leq\left.(2 \pi)^{v}|| f\right|_{v+1} ^{v+1}<\infty,
\end{aligned}
$$

from which we have that $\mathcal{G}_{f}^{v}$ is bounded above. Notice also that if $f \equiv \frac{1}{2 \pi}$ and $X$ is a finite measure space with $\mu(X)=2^{v+1} \pi \Gamma(v+1)$, then $\mathcal{G}_{f}^{v}(x)=g_{v}(x)$ for $x>0$.

In the remaining part of this paper we clarify how this class of functions came into being and how it is related to some other well-known functions.

\section{MAIN RESULT}

Functions are central objects in most fields of mathematics and science. One of the most important tools that we use to investigate the size of these objects, not its point-wise behavior is a function itself which is known as the distribution function. In the following, we review the main tools that we shall use to conclude our main result, see [3,7-9].

We start by recalling the definition of the distribution function.

Definition 1. Let $(X, \mu)$ be a $\sigma$-finite measure space and $f: X \rightarrow \mathbb{C}$ be a measurable function. The distribution function $d_{f}$ is given by

$$
d_{f}(\alpha)=\mu\{x \in X:|f(x)|>\alpha\},
$$

where $\alpha \geq 0$. 
If $f \in L^{p}(X, \mu)$ for $0<p<\infty$, then we have the following integral formula which correlates between the $L^{p}$-norm of $f$ and its distribution function.

$$
\|f\|_{L^{p}}^{p}=p \int_{0}^{\infty} d_{f}(\alpha) \alpha^{p-1} \mathrm{~d} \alpha .
$$

We know that if $\eta$ is a radial function, then

$$
\int_{\mathbb{R}^{n}} \eta(x) \mathrm{d} x=\sigma\left(\mathbb{S}^{n-1}\right) \int_{0}^{\infty} \eta(r) r^{n-1} \mathrm{~d} r,
$$

where $\sigma\left(\mathbb{S}^{n-1}\right)=\frac{2 \pi^{n / 2}}{\Gamma(n / 2)}$. Thus, in view of (2.1) and (2.2) we can think about $d_{f}$ as an integrable radial function on $\mathbb{R}^{p}$. To be more precise, we have

$$
\|f\|_{L^{p}}^{p}=\frac{p}{\sigma\left(\mathbb{S}^{p-1}\right)} \int_{\mathbb{R}^{p}} d_{f}(|y|) \mathrm{d} y .
$$

Equation (2.3) says that $d_{f} \in L^{1}\left(\mathbb{R}^{p}\right)$ whenever $f \in L^{p}(X, \mu)$ and so its Fourier transform exists. The following result shows how the class $\mathcal{G}_{f}^{v}$ did arise.

Theorem 1. Let $X$ be a $\sigma$-finite measure space and $f \in L^{2}(X, \mu)$. Then $\mathcal{G}_{f}^{1}(\xi)=$ $\hat{d}_{f}(\xi), \xi \in \mathbb{R}^{2}$.

Proof. By using the fact that the Fourier transform of a radial function $\eta$ is given by

$$
\hat{\eta}(\xi)=\frac{2 \pi}{|\xi|^{\frac{n-2}{2}}} \int_{0}^{\infty} \eta(r) J_{\frac{n}{2}-1}(2 \pi r|\xi|) r^{\frac{n}{2}} \mathrm{~d} r
$$

we have

$$
\begin{aligned}
\hat{d}_{f}(\xi) & =\int_{\mathbb{R}^{2}} e^{-2 \pi i y \cdot \xi} d_{f}(|y|) \mathrm{d} y \\
& =2 \pi \int_{0}^{\infty} d_{f}(r) J_{0}(2 \pi r|\xi|) r \mathrm{~d} r .
\end{aligned}
$$

The definition of distribution function and (2.4) give

$$
\begin{aligned}
\hat{d}_{f}(\xi) & =2 \pi \int_{0}^{\infty} m\{x \in X:|f(x)|>r\} J_{0}(2 \pi r|\xi|) r \mathrm{~d} r \\
& =2 \pi \int_{0}^{\infty}\left(\int_{\{z \in X:|f(z)|>r\}} \mathrm{d} \mu\right) J_{0}(2 \pi r|\xi|) r \mathrm{~d} r .
\end{aligned}
$$

Noting that $\mathbb{1}_{\{z \in X:|f(z)|>r\}}(x) \equiv \mathbb{1}_{(0,|f(x)|)}(r)$ and applying Fubini's theorem we obtain

$$
\hat{d}_{f}(\xi)=2 \pi \int_{0}^{\infty}\left(\int_{X} \mathbb{1}_{\{z \in X:|f(z)|>r\}}(x) \mathrm{d} \mu(x)\right) J_{0}(2 \pi r|\xi|) r \mathrm{~d} r
$$




$$
\begin{aligned}
& =2 \pi \int_{X}\left(\int_{0}^{\infty} \mathbb{1}_{(0,|f(x)|)}(r) J_{0}(2 \pi r|\xi|) r \mathrm{~d} r\right) \mathrm{d} \mu(x) \\
& =2 \pi \int_{X}\left(\int_{0}^{|f(x)|} J_{0}(2 \pi r|\xi|) r \mathrm{~d} r\right) \mathrm{d} \mu(x) .
\end{aligned}
$$

Now, by using the change of variables, $s=r /|f(x)|$, we have

$$
\hat{d}_{f}(\xi)=2 \pi \int_{X}|f(x)|^{2}\left(\int_{0}^{1} J_{0}(2 \pi s|f(x)||\xi|) s \mathrm{~d} s\right) \mathrm{d} \mu(x) .
$$

Next, applying the integral identity

$$
\int_{0}^{1} J_{\alpha}(t s) s^{\alpha+1}\left(1-s^{2}\right)^{\beta} \mathrm{d} s=\frac{\Gamma(\beta+1) 2^{\beta}}{t^{\beta+1}} J_{\alpha+\beta+1}(t)
$$

with $\alpha=\beta=0$, we get

$$
\begin{aligned}
\hat{d}_{f}(\xi) & =2 \pi \int_{X}|f(x)|^{2}\left(\frac{1}{2 \pi|f(x)||\xi|} J_{1}(2 \pi|f(x)||\xi|)\right) \mathrm{d} \mu(x) \\
& =\frac{1}{|\xi|} \int_{X}|f(x)| J_{1}(2 \pi|f(x)||\xi|) \mathrm{d} \mu(x) \\
& =\mathcal{G}_{f}^{1}(\xi) .
\end{aligned}
$$

Remark 1 . If $p$ is an integer greater than 2 , then using the integral identity

$$
\int t^{v} J_{v-1}(t) \mathrm{d} t=t^{v} J_{v}(t)+C
$$

in place of (2.8) and applying the same technique that was used in the proof of Theorem 1 we have $\mathcal{G}_{f}^{p / 2}(\xi)=\hat{d}_{f}(\xi), \xi \in \mathbb{R}^{p}$.

Remark 2. Theorem 1 and Remark 1 show that $\mathcal{G}_{f}^{p / 2}(\xi)$ enjoys the property that it is uniformly continuous on $\mathbb{R}^{p}$. Moreover, $\mathcal{G}_{f}^{p / 2}(\xi) \rightarrow 0$ as $|\xi| \rightarrow \infty$.

Theorem 2. If $X$ is a finite measure space and $f \in L^{2}(X, \mu)$, then $\mathcal{G}_{f}^{1} \in L^{2}\left(\mathbb{R}^{2}\right)$.

Proof. Notice that

$$
\begin{aligned}
\left(\int_{\mathbb{R}^{2}}\left|\mathcal{G}_{f}^{1}(\xi)\right|^{2} \mathrm{~d} \xi\right)^{1 / 2} & =\left(\int_{|\xi|<1}\left|\mathcal{G}_{f}^{1}(\xi)\right|^{2} \mathrm{~d} \xi+\int_{|\xi|>1}\left|\mathcal{G}_{f}^{1}(\xi)\right|^{2} \mathrm{~d} \xi\right)^{1 / 2} \\
& =\left(\int_{|\xi|<1}\left|\mathcal{G}_{f}^{1}(\xi)\right|^{2} \mathrm{~d} \xi\right)^{1 / 2}+\left(\int_{|\xi|>1}\left|\mathcal{G}_{f}^{1}(\xi)\right|^{2} \mathrm{~d} \xi\right)^{1 / 2} \\
& :=I+I I .
\end{aligned}
$$


By using (1.1), we have

$$
\begin{aligned}
I & =\left(\int_{|\xi|<1}\left|\mathcal{G}_{f}^{1}(\xi)\right|^{2} \mathrm{~d} \xi\right)^{1 / 2} \\
& \leq\left(\int_{|\xi|<1}\left(\frac{1}{|\xi|} \int_{X}|f(x)|\left|J_{1}(2 \pi|f(x)||\xi|)\right| \mathrm{d} \mu(x)\right)^{2} \mathrm{~d} \xi\right)^{1 / 2} \\
& \leq 2 \pi\left(\int_{|\xi|<1}\left(\frac{1}{|\xi|} \int_{X}|f(x)|^{2}|\xi| \mathrm{d} \mu(x)\right)^{2} \mathrm{~d} \xi\right)^{1 / 2} \\
& \leq 2 \pi^{3 / 2}|| f \|_{L^{2}(X, \mu)} .
\end{aligned}
$$

Again, applying (1.2) we obtain

$$
\begin{aligned}
I I & =\left(\int_{|\xi|>1}\left|\mathcal{G}_{f}^{1}(\xi)\right|^{2} \mathrm{~d} \xi\right)^{1 / 2} \\
& \leq\left(\int_{|\xi|>1}\left(\frac{1}{|\xi|} \int_{X}|f(x)|\left|J_{1}(2 \pi|f(x)||\xi|)\right| \mathrm{d} \mu(x)\right)^{2} \mathrm{~d} \xi\right)^{1 / 2} \\
& \leq \frac{1}{\sqrt{2 \pi}}\left(\int_{|\xi|>1} \frac{1}{|\xi|^{3}}\left(\int_{X}|f(x)|^{1 / 2} \mathrm{~d} \mu(x)\right)^{2} \mathrm{~d} \xi\right)^{1 / 2} \\
& \leq \frac{1}{\sqrt{2 \pi}}\left(\int_{X}|f(x)|^{1 / 2} \mathrm{~d} \mu(x)\right)\left(\int_{|\xi|>1} \frac{1}{|\xi|^{3}} \mathrm{~d} \xi\right)^{1 / 2} \\
& =(\mu(X))^{3 / 4} \sqrt{|| f \|_{L^{2}(X, \mu)}} .
\end{aligned}
$$

Now, combining (2.10), (2.11) and (2.12) we get the desired result.

\section{CONCLUSION}

We have introduced a new class of functions which are defined via integrals. We have proved that the function $\mathcal{G}_{f}^{v}$ is uniformly continuous and can be an element of the Lebesgue spaces for some values of $v$. Fourier analysis techniques were used to show that Bessel functions are related to the statistical terminology "distribution function". The main findings of this paper should open the door for studying these functions extensively.

\section{REFERENCES}

[1] M. Abramowitz and I. Stegun, Handbook of Mathematical Functions with Formulas, Graphs, and Mathematical Tables. New York: Dover Publications, Inc., 1965.

[2] R. Askey, "Grünbaum's inequality for Bessel functions." J. Math. Anal. Appl., vol. 41, pp. 122-124, 1973.

[3] L. Grafakos, Classical Fourier Analysis. New York: Springer-Verlag New York, 2014. 
[4] F. Grünbaum, "A property of Legendre polynomials." Proc. Nat. Acad. Sci., USA., vol. 67, pp. 959-960, 1970.

[5] E. Neuman, "Inequalities involving Bessel functions of the first kind." J. Ineq. Pure Appl. Math., vol. 5 , no. 4 , pp. 1-4, 2004.

[6] F. W. J. Olver, , D. W. Lozier, R. F. Boisvert, and C. W. Clark, The NIST Handbook of Mathematical Functions. Cambridge Univ. Press, 2010.

[7] H. Royden, P. Fitzpatrick, and H. Royden, Real analysis. Boston: Prentice Hall, 2010.

[8] E. Stein and G. Weiss, Introduction to Fourier Analysis on Euclidean Spaces (PMS-32). New Jersey: Princeton University Press, 1971.

[9] G. Watson, A Treatise on the Theory of Bessel Functions. Cambridge: Cambridge University Press, 1966.

Authors' addresses

H. Laith

German Jordanian University, Mathematics Department, Amman, Madaba, 11180, Jordan

E-mail address: laith.hawawsheh@gju.edu. jo

\section{A. Mohammad}

German Jordanian University, Mathematics Department, Amman, Madaba, 11180, Jordan

E-mail address: mohammad.abudayah@g ju. edu. jo

H. Dutta

Gauhati University, Mathematics Department, Guwahati, 781014 Assam, India

E-mail address: hemen_dutta08@rediffmail.com 